\title{
Estado nutricional de adultos entre 20 e 59 anos segundo os indicadores do sistema de vigilância alimentar e nutricional (Sisvan) na atenção básica
}

\author{
Adult nutritional state between 20 and 59 years old according to the food and nutritional \\ surveillance system indicators (Sisvan) in basic care \\ Estado nutricional del adulto de 20 Y 59 años según los indicadores del sistema de vigilancia \\ alimentaria y nutricional (Sisvan) en cuidados básicos
}

Recebido: 26/04/2021 | Revisado: 05/05/2021 | Aceito: 11/05/2021 | Publicado: 30/05/2021

Rhayana Marques dos Santos ORCID: https://orcid.org/0000-0001-8820-091X Faculdade de Ciências Humanas de Olinda, Brasil E-mail: marquesrhayana@hotmail.com

Fabiana Santos da Silva Oliveira ORCID: https://orcid.org/0000-0002-9686-3657 Faculdade de Ciências Humanas de Olinda, Brasil E-mail: fabiana_san@hotmail.com

Luana Yasmin Araujo Costa

ORCID: https://orcid.org/0000-0001-7968-0392 Centro Universitário dos Guararapes, Brasil

E-mail: luanayasminnutricionista@gmail.com

Vania Maria Silva de Moraes

ORCID: https://orcid.org/0000-0002-8627-1690 Faculdade de ciências Humanas de Olinda, Brasil E-mail: vaniaenf_moraes@hotmail.com

Gleyce Kelly de Araujo

ORCID: https://orcid.org/0000-0002-9388-7389 Universidade Federal de Pernambuco, Brasil

E-mail: gleycearaujo.nutri@gmail.com

Maria Suzane da Silva Barbosa

ORCID: https://orcid.org/0000-0003-1995-8721

Universidade Federal de Pernambuco, Brasil

E-mail: m.suzane.barbosa@gmail.com

Silvia Mariana da Silva Barbosa

ORCID: https://orcid.org/0000-0001-5252-9705

Universidade Federal de Pernambuco, Brasi

E-mail: barbosasms@gmail.com

Flavia Maria Barros Lavra

ORCID: https://orcid.org/0000-0002-4299-7529

Faculdade de Ciências Humanas de Olinda, Brasil

E-mail: proflavialavra@hotmail.com

Fabio Torres Cunha

ORCID: http://orcid.org/0000-0003-0238-7600 Faculdade de Comunicação, Tecnologia e Turismo de Olinda, Brasil

E-mail: fabiologo1980@gmail.com

Danielle Cássia de Oliveira

ORCID: https://orcid.org/0000-0003-4154-2597

Faculdade Ciências Humanas de Olinda, Brasil

E-mail: daniellecassiao@yahoo.com.br

\section{Resumo}

Objetivo: Identificar o estado nutricional de adultos entre 20 a 59 anos na Atenção Primária à Saúde. Metodologia: trata-se de um estudo descritivo, transversal, com abordagem quantitativa. Realizado a partir de relatórios públicos online disponíveis no Sistema de Vigilância Alimentar e Nutricional (SISVAN). Onde foram extraídos os dados nutricionais dos adultos de 20 a 59 anos, atendidos na Atenção Primária à Saúde, nas cinco regiões do Brasil, no período de 2015 a 2019. Resultados: O estudo apresentou que o Nordeste se destaca com o maior percentual de Baixo Peso, a região Norte com maior percentual de Peso Adequado e Sobrepeso, e a Região Sul apresentou maior índice de Obesidade ao longo desses cinco anos. Considerações finais: A partir disso, espera-se que a tabulação, o 
acompanhamento e a intervenção nutricional dos adultos atendidos na Atenção Primária a Saúde seja contínuo para que se tenha o aumento da expectativa de vida, resultando em um envelhecimento saudável da população brasileira.

Palavras-chave: Desnutrição; Atenção básica; Políticas públicas; Sobrepeso.

\begin{abstract}
Objective: Identify the nutritional status of adults between 20 and 59 years old in Primary Health Care. Methodology: this is a descriptive, cross-sectional study, with a quantitative approach. Conducted from online public reports available in the Food and Nutrition Surveillance System (SISVAN). Were extracted the nutritional data of adults aged from 20 to 59, attended in Primary Health Care in the five regions of Brazil, in the period 2015-2019. Results: The study showed that the Northeast stands out with the highest percentage of low weight, the northern region with the highest percentage of weight Adequate and overweight, and the South Region presented the highest obesity rate over these five years. Final considerations: From this, it is expected that the tabulation, monitoring and nutritional intervention of adults assisted in Primary Health Care will be continuous so that there is an increase in life expectancy, resulting in a healthy aging of the Brazilian population.
\end{abstract}

Keywords: Malnutrition; Basic care; Public policy; Overweight.

\title{
Resumen
}

Objetivo: identificar el estado nutricional de adultos entre 20 y 59 años en Atención Primaria de Salud. Metodología: se trata de un estudio descriptivo, transversal, con enfoque cuantitativo. Elaborado a partir de informes públicos en línea disponibles en el Sistema de Vigilancia Alimentaria y Nutricional (SISVAN). Donde se extrajeron los datos nutricionales de adultos de 20 a 59 años, atendidos en Atención Primaria de Salud, en las cinco regiones de Brasil, en el período de 2015 a 2019. Resultados: El estudio mostró que el Noreste se destaca con el mayor porcentaje de Bajo Peso, la Región Norte con el mayor porcentaje de Adecuado y Sobrepeso, y la Región Sur presentó el mayor índice de Obesidad en estos cinco años. Consideraciones finales: A partir de esto, se espera que la tabulación, seguimiento e intervención nutricional de los adultos atendidos en Atención Primaria de Salud sea continua para que haya un aumento de la esperanza de vida, resultando en un envejecimiento saludable de la población brasileña.

Palabras clave: Desnutrición; Cuidado básico; Políticas públicas; Exceso de peso.

\section{Introdução}

O estado nutricional é a combinação e o equilíbrio entre a ingesta alimentar, as necessidades nutricionais, e do estado de saúde, que está diretamente associado a fatores causais sendo a nível familiar, ambiental e demográfico, podendo vir a ser influenciado por outros fatores como o aspecto social e econômico. A alimentação é substancial para o crescimento, desenvolvimento e qualidade de vida do ser humano, sendo um dos determinantes e condicionantes da saúde, é um direito pertinente a todas as pessoas (Mazur \& Navarro, 2015).

Nas últimas décadas a população mundial vem sofrendo grandes alterações em seu perfil de morbimortalidade, marcadas principalmente pelo aumento importante da prevalência de indivíduos com sobrepeso e obesidade, e a elevação relativa de óbitos decorrentes de doenças crônicas não transmissíveis (DCNT) como diabetes mellitus (DM), hipertensão arterial sistêmica (HAS) além de alguns tipos de câncer (Asakura et al., 2015).

Além disso, pesquisas atuais apresentaram transição alimentar na população brasileira, em especial da população adulta, que vem revelando atualmente um avanço epidêmico de excesso de peso, seguindo a tendência mundial. Vale ressaltar que tais práticas podem causar diversos agravos, necessitando medidas urgentes de promoção e prevenção, a partir do monitoramento nutricional regular (Silva et al., 2015).

De acordo com dados da Pesquisa Nacional de Saúde (2020), em comparação com os últimos resultados, em 2019 os adultos de 20 anos ou mais de idade, apresentaram uma prevalência no excesso de peso (60,0\% nos homens e $63,3 \%$ nas mulheres) e obesidade (22,8\% nos homens e $30,2 \%$ nas mulheres).

Com base nisto, no Brasil foram implantadas políticas públicas, dentre as quais surgiu a Política Nacional de Alimentação e Nutrição (PNAN), que visa à melhoria das condições de alimentação, nutrição e saúde da população. Para o alcance no propósito de melhoria alimentar, o PNAN tem como uma de suas diretrizes a Vigilância Alimentar e Nutricional 
(VAN), que utiliza um sistema de informação online próprio, denominado de Sistema de Vigilância Alimentar e Nutricional (SISVAN). Tal componente vem trazendo dados sobre as condições nutricionais que a população brasileira assistida pelo SUS se encontra (Brasil, 2015).

O SUS dispõe da Atenção Primária como sua porta de entrada, tendo como principal objetivo: acolher, escutar e oferecer respostas resolutivas para a maioria dos problemas de saúde da população, minimizando seus danos e sofrimentos (Souza, 2016). Sendo assim, as equipes de saúde da família possuem um papel fundamental na captação e inclusão dos dados nutricionais, que são incluídos no SISVAN. Sistema esse que apresenta informações do Índice de Massa Corporal - IMC, originados do peso e altura coletados. Tais informações classificam o estado nutricional do indivíduo que é a base do sistema. Além disso, é de responsabilidade das equipes de Atenção Básica a promoção da alimentação saudável, além de acompanhamento e monitoramento individual, ainda podendo encaminhá-lo para uma equipe especializada da Rede de Atenção à Saúde (Nascimento, Silva \& Jaime, 2017).

Diante dos agravos com o aumento de doenças crônicas não transmissíveis (DCNT), associado a mudanças no padrão de consumo alimentar e ao estilo de vida apresentado pela população mundial, faz-se necessário iniciativas científicas, que identifiquem o diagnóstico nutricional da população no qual a partir de sua avaliação sistemática seja possível à promoção de atividades para a população de risco, onde os dados em que apresentam o sobrepeso e a obesidade embasam maior relevância para as ações. Em razão disso, o presente estudo vale de extrema importância com o seu conteúdo para montar estratégias de intervenção, favorecendo assim, o aperfeiçoamento da assistência em saúde.

A partir do que foi dito, o objetivo deste estudo é identificar o estado nutricional de adultos brasileiros entre 20 a 59 anos na Atenção Primária à Saúde.

\section{Metodologia}

Este estudo é do tipo descritivo, transversal, visando expor fatos e características presentes em uma determinada população ou área de interesse, com abordagem quantitativa, fazendo a quantificação dos dados obtidos (Martins, 2015). Foi realizado a partir de relatórios públicos online dos estados nutricionais disponíveis no Sistema de Vigilância Alimentar e Nutricional (SISVAN). Tal sistema concede os dados sobre o consumo alimentar e o estado nutricional de todas as faixas etárias da população atendida na Atenção Básica, onde tais dados são cadastrados pelos gestores da Unidade de Saúde.

Foram analisados os dados nutricionais de todos os registros disponíveis no SISVAN WEB, dos adultos, de ambos os sexos, dos 20 a 59 anos, atendidos na Atenção Primária a Saúde, nas cinco regiões brasileiras (Norte, Nordeste, Centro-oeste, Sul e Sudeste), no período de 2015 a 2019, logo após, foram registrados em planilha do Excel, tabulados em percentis e classificados segundo a OMS (1998), sendo Baixo Peso $\left(<18,5 \mathrm{~kg} / \mathrm{m}^{2}\right)$, Peso Adequado ou Eutrófico $\left(\geq 18,5 \mathrm{~kg} / \mathrm{m}^{2}\right.$ e $<25$ $\left.\mathrm{kg} / \mathrm{m}^{2}\right)$, Sobrepeso $\left(\geq 25 \mathrm{~kg} / \mathrm{m}^{2} \mathrm{a}<30 \mathrm{~kg} / \mathrm{m}^{2}\right)$, e Obesidade $\left(\geq 30 \mathrm{~kg} / \mathrm{m}^{2}\right)$, no qual subdivide-se em grau I $\left(\geq 30 \mathrm{~kg} / \mathrm{m}^{2} \mathrm{e}<\right.$ $\left.35 \mathrm{~kg} / \mathrm{m}^{2}\right)$, grau II $\left(\geq 35 \mathrm{~kg} / \mathrm{m}^{2} \mathrm{e}<40 \mathrm{~kg} / \mathrm{m}^{2}\right)$, e grau III $\left(\geq 40 \mathrm{~kg} / \mathrm{m}^{2}\right)$, onde os dados da obesidade serão agrupados em uma única classificação. A coleta de dados foi realizada no período de maio a junho de 2020.

Segundo resolução no 466, de 12 de dezembro de 2012 do conselho nacional de saúde, não há necessidade de comitê de ética porque os resultados já estão disponíveis no site do SISVAN para consulta pública.

\section{Resultados}

De acordo com os dados disponíveis no SISVAN-WEB da população atendida na Atenção Primária à Saúde de todas as regiões brasileiras nos anos de 2015 a 2019 podemos constatar que o Nordeste se destaca com o maior percentual de Baixo 
Peso, a região Norte com maior percentual de Peso Adequado e Sobrepeso, e a Região Sul apresentou maior índice de Obesidade ao longo desses cinco anos conforme podemos verificar nos gráficos a seguir.

No Gráfico 1, o Nordeste se destacou com o percentual mais elevado de Baixo Peso na maioria dos anos, exceto em 2017, onde é levemente ultrapassado pelo Centro-Oeste. O Nordeste apresentou percentual de 2,24\%, 2,32\%, 2,40\%, 2,32\%, e $2,34 \%$ respectivamente.

Gráfico 1 - Dados disponíveis do estado nutricional de adultos (20 a 59 anos) das regiões brasileiras em relação ao indicador Baixo Peso, Recife-PE.

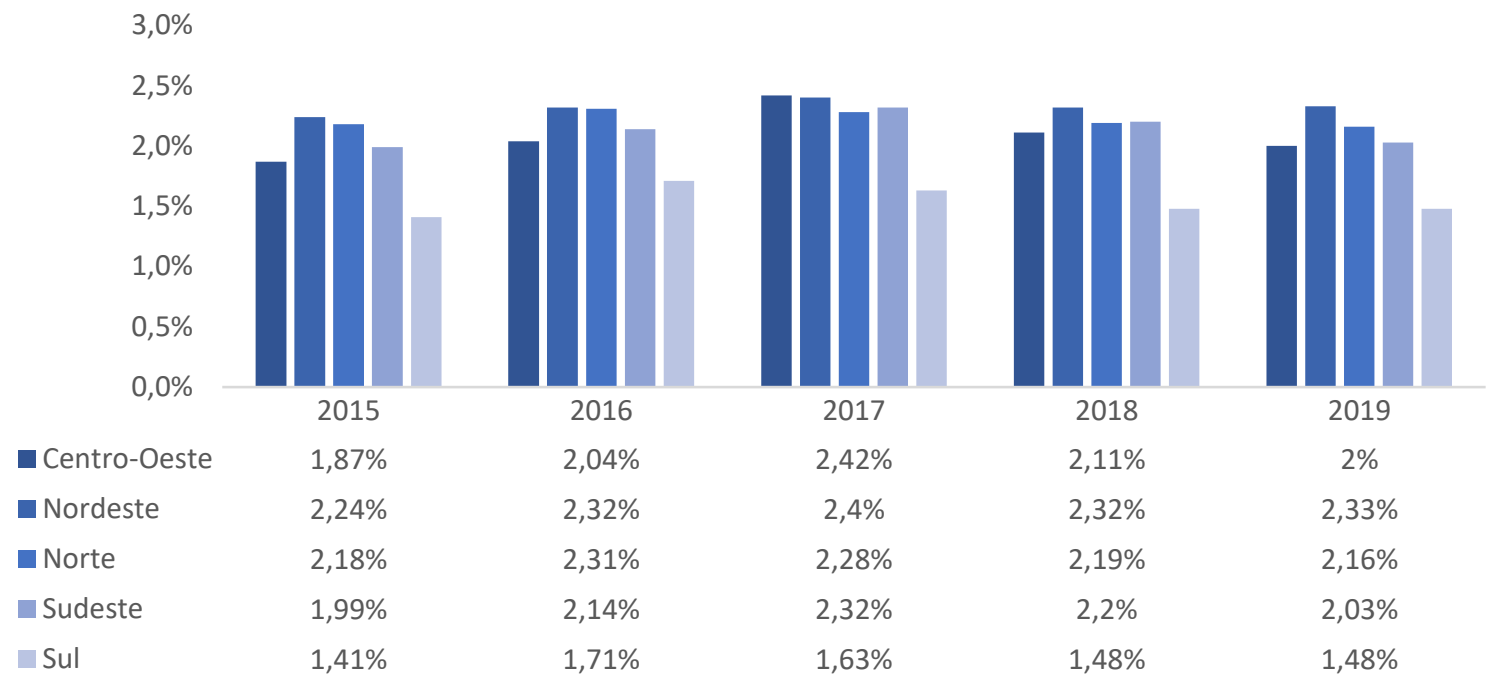

*Fonte: Dados obtidos do SISVAN-WEB (2020).

De acordo com o Gráfico 2 que mostra o percentual do Peso Adequado, a região Norte se destacou nos anos de 2016 a 2019 , onde apresentou um percentual de 35,15\%,36,44\%, 35,96\%, 34,98\%, 34,15\% respectivamente. Sendo ultrapassado apenas em 2015 pela região Nordeste, que apresentou o percentual mais elevado das regiões.

Gráfico 2 - Dados disponíveis do estado nutricional de adultos (20 a 59 anos) das regiões brasileiras em relação ao indicador Peso Adequado, Recife-PE.

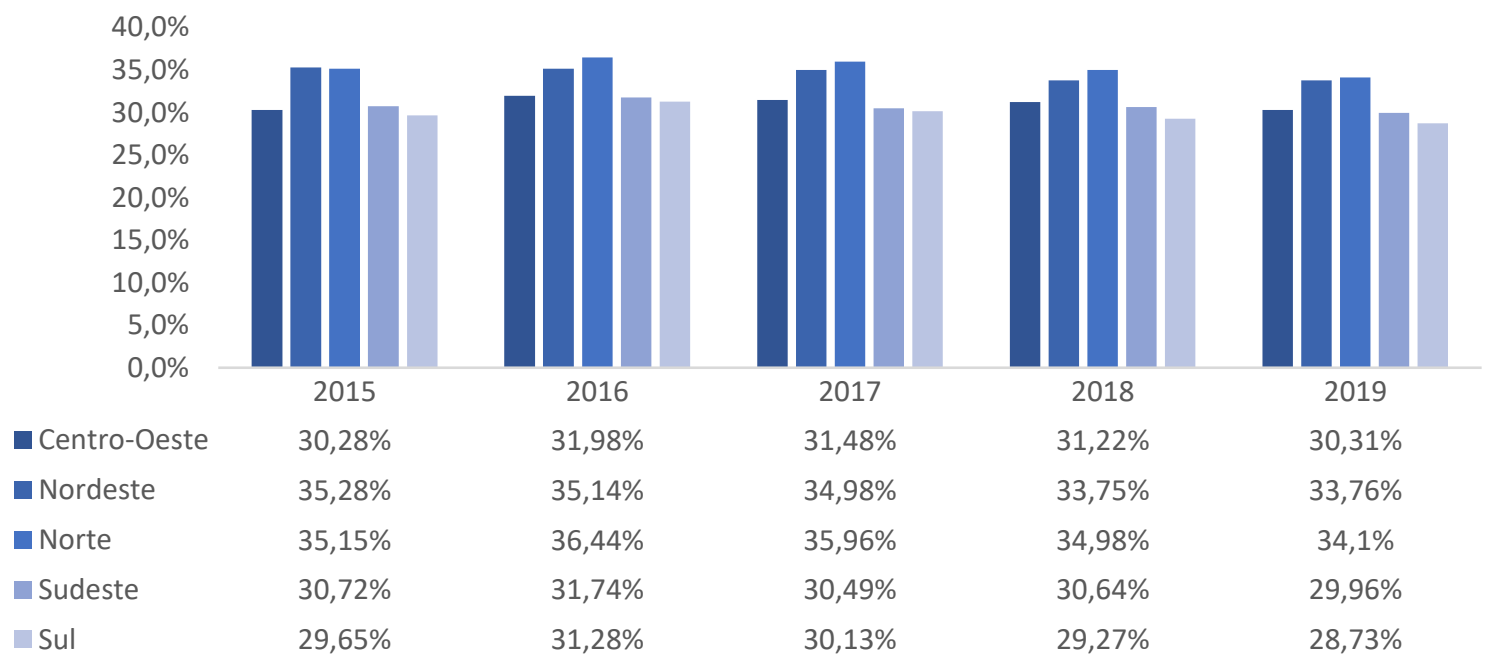


*Fonte: Dados obtidos do SISVAN-WEB (2020).

No Gráfico 3 do Sobrepeso, a região Norte apresentou o maior índice nos últimos três anos, indicando um percentual de $36,36 \%, 36,60 \%$, e 36,80\% respectivamente, sendo ultrapassado em 2015 e 2016 pela região Nordeste.

Gráfico 3 - Dados disponíveis do estado nutricional de adultos (20 a 59 anos) das regiões brasileiras em relação ao indicador Sobrepeso, Recife-PE.

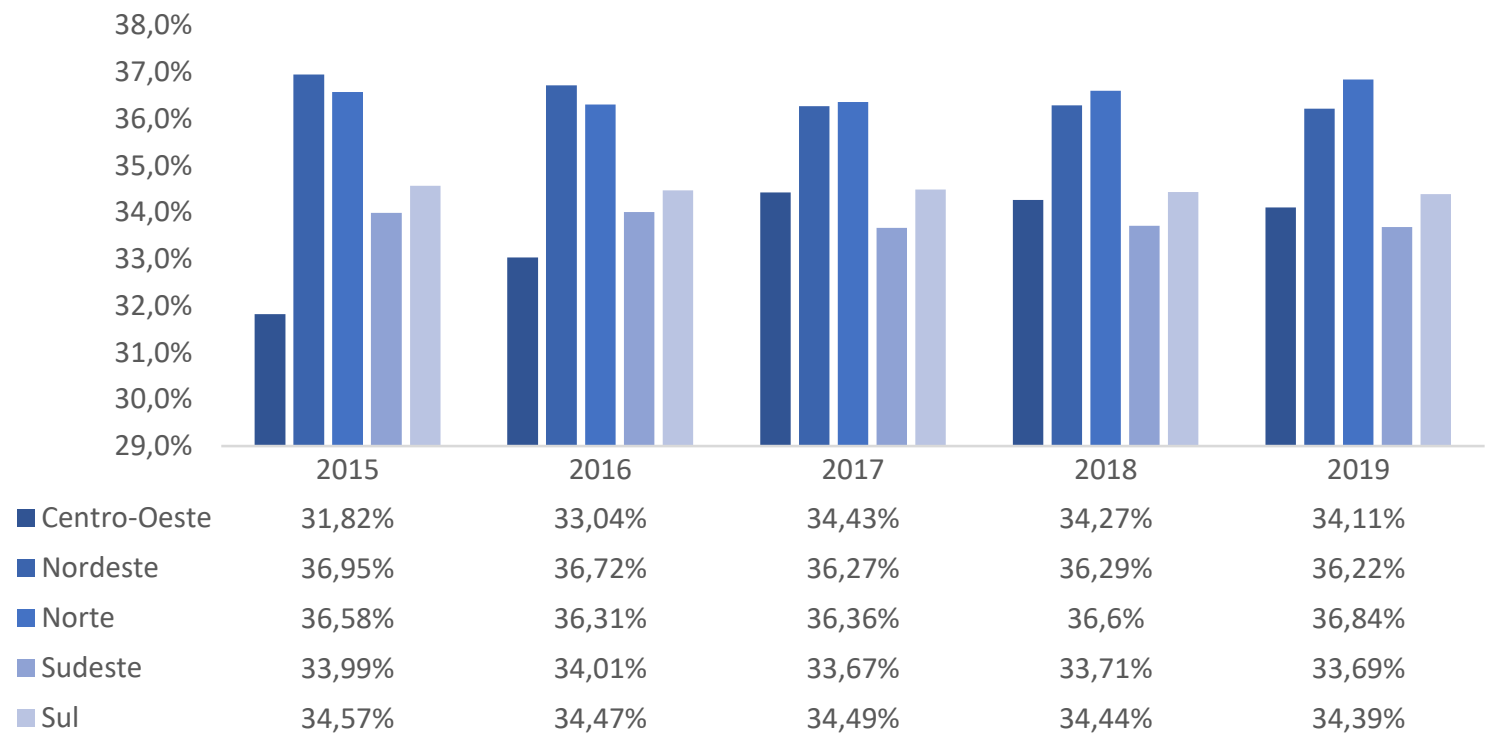

*Fonte: Dados obtidos do SISVAN-WEB (2020).

No Gráfico 4, a obesidade na região Sul apresentou uma elevação no decorrer dos anos de 2017 a 2019, revelando um percentual ascendente de 33,76\%,34,80\% e 35,48\%, respectivamente. A região Sul foi ultrapassada pelo Centro-Oeste nos anos de 2015 e 2016.

Gráfico 4 - Dados disponíveis do estado nutricional de adultos (20 a 59 anos) das regiões brasileiras em relação ao indicador Obesidade, Recife-PE.

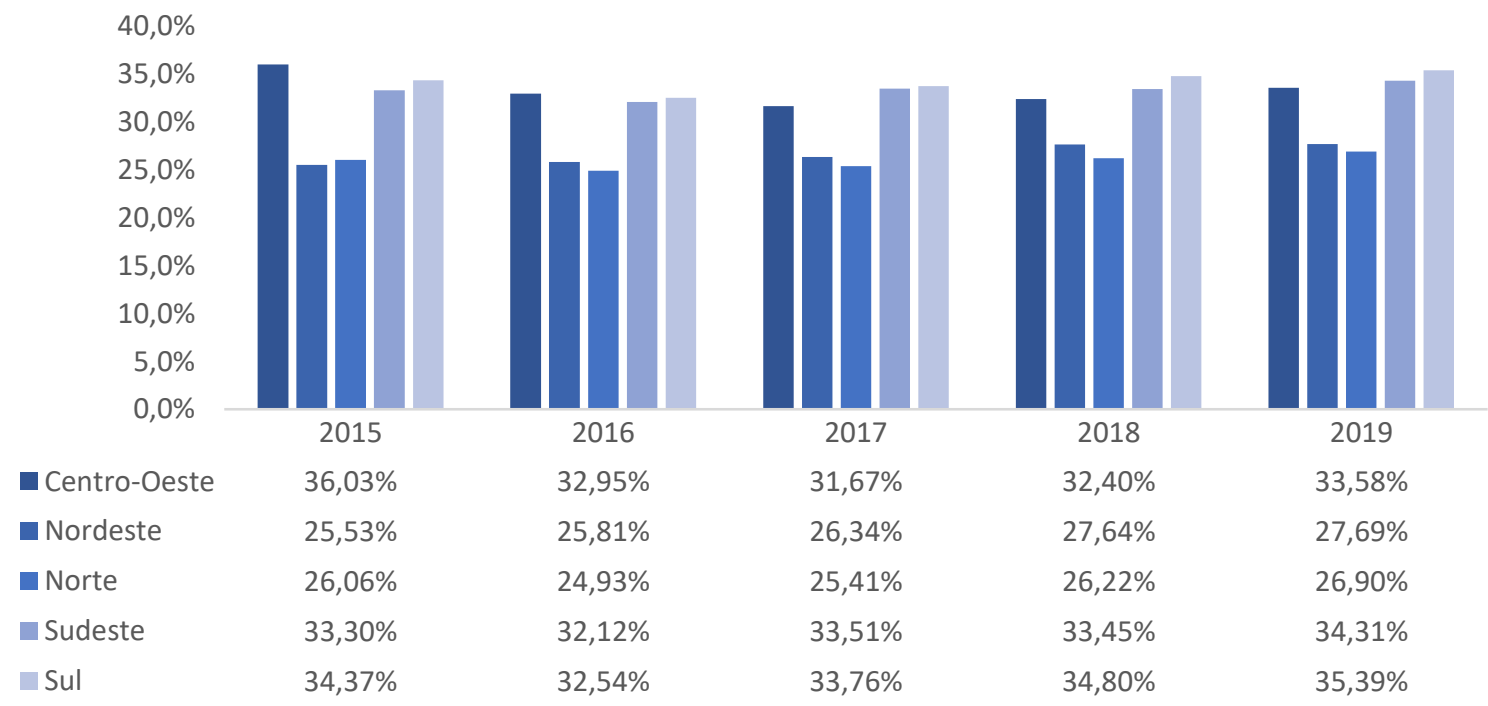




\section{Discussão}

*Fonte: Dados obtidos do SISVAN-WEB (2020).

Diante dos achados obtidos neste estudo, podemos compreender que o Brasil é um país extenso em seu território, e que cada região possui suas características individuais, e que inúmeros fatores podem contribuir para os desvios nutricionais apresentados em cada região.

O IBGE apresentou na Pesquisa de Orçamentos Familiares (POF) (2020), que há desigualdades entre as regiões brasileiras, onde há uma elevada disparidade entre as áreas urbanas e rurais.

Foi percebido que de todas as regiões o Nordeste é a mais afetada com o Baixo Peso, na maioria dos anos. Lourenço, et. al., (2019), destaca que o indivíduo adulto pode trazer de sua infância sequelas da desnutrição infantil que contribui para enfermidades que perduram para toda a vida.

Souza, et. al. (2018), ressalta em seu estudo que os avanços que aconteceram ao longo das três décadas da implementação dos SUS no Brasil, onde ocorreram ações de intervenção na desnutrição, como o aumento do aleitamento materno, manejo da diarreia, decorreram principalmente pela expansão da APS com a Estratégias de Saúde da Família- ESF, e rede de pré-atendimento hospitalar, com o aumento da cobertura assistencial da população, em especial as mais vulneráveis, com acesso gratuito a medicamentos, ampliação do calendário vacinal, e também a melhoria do saneamento básico onde apresentou uma importante redução nas doenças infecto-parasitárias.

No aspecto econômico, podemos destacar que o Nordeste tem o menor rendimento familiar de todas as regiões, tendo um elevado índice de aposentadorias e pensões do INSS e uma extensa participação nos programas sociais federais (IBGE, 2020).

Pode-se destacar também que um baixo Índice de Desenvolvimento Humano - IDH associado aos fatores socioeconômicos, ambientais e de insegurança alimentar estão presentes em grande parte dos estados da região Nordeste (Cabral, et. al., 2013).

Há também um aumento do consumo de alimentos fora do lar atualmente, que contribui para o consumo inadequado ou insuficiente de energia e nutrientes, com a ingesta de proteínas, fibra alimentar e ferro consideravelmente inferior se comparados aos que se alimentavam em seu lar, onde a área urbana é a que mais se destaca (Cavalcante, et. al., 2017).

Embora o problema de má nutrição na região Nordeste tenha melhorado ao longo dos anos, resultante do ganho econômico, da ampliação dos programas governamentais, como de maior acesso à saúde e de transferência de renda, ainda podemos observar que a desnutrição ainda resiste discretamente (Cabral, et. al., 2013).

O Norte se sobressaiu como a região que tem o maior percentual de Peso Adequado e Sobrepeso comparado às demais regiões.

Segundo a POF realizada pelo IBGE em 2020, o consumo de alimentos naturais e minimamente processados é maior no meio rural comparado com o meio urbano, onde a maior disponibilidade desses alimentos é no Norte e Nordeste, no meio rural e entre as famílias de menor renda.

Os alimentos naturais ou minimamente processados que se destacaram no Norte foram a farinha de mandioca, carnes, vísceras e pescados. No Nordeste o feijão, farinha de milho e o fubá. O açúcar e os pães no Norte e Nordeste tiveram um consumo baixo, com exceção de biscoitos salgados e margarina que foram maiores no Nordeste (IBGE, 2020).

Vale ressaltar que após o compromisso firmado pelo Estado Brasileiro com o SUS onde se pactua políticas públicas, como a Política Nacional de Segurança Alimentar e Nutricional, que visam o combate à fome e a diminuição da pobreza e da desigualdade, houve uma melhoria na qualidade de vida. O Pacto Nacional para Alimentação Saudável de 2017 surgiu com esse objetivo, de trazer oferta, disponibilidade e consumo de alimentos saudáveis para os cidadãos brasileiros. 
A região Sul vem apresentando os maiores índices de obesidade. Os alimentos que se destacam no consumo da região Sul são a farinha de trigo e os queijos e predominam os alimentos ultraprocessados (Vigitel Brasil, 2020). Essa região apresenta o menor percentual de consumo de cereais, leguminosas e oleaginosas (IBGE 2020).

Vedana, em (2008), já apontava que a região Sul era a que possuía o maior índice de obesidade entre as regiões, igualando-se ou até ultrapassando os países desenvolvidos.

Segundo Mazur e Navarro (2015), atualmente o número de pessoas obesas ultrapassam o de desnutridos, onde podemos perceber a partir dos gráficos deste estudo. Mundialmente, em 2008, mais de 1,4 bilhões de adultos estavam acima do peso, onde mais de 200 milhões eram homens e quase 300 milhões eram mulheres.

De acordo com pesquisa atual sobre insegurança alimentar e obesidade, repara-se que as mulheres lideram o percentual de sobrepeso e obesidade, e que a sua maioria são pardas ou negras, possuem baixa escolaridade, rendimento domiciliar de até um salário mínimo, com quatro ou cinco moradores em sua residência (Soares, et al., 2020).

Desde de 1980, as taxas de obesidade vêm crescendo mundialmente, onde pesquisas recentes associam o aumento de doenças, e de outros fatores, até ao consumo insuficiente de micronutrientes essenciais (cálcio, ferro, vitaminas A, C, D e E) (Cembranel, et al., 2017).

Segundo Souza, et. al., (2018), 75\% das mortes no Brasil são lideradas pelas DCNT, e são causadas principalmente pelas doenças cardiovasculares, doenças respiratórias, e por complicações do diabetes. A doença isquêmica do coração permaneceu em primeiro lugar no ranking das principais causas de morte de 1990 a 2015, seguida pela doença cerebrovascular, a Doença Pulmonar Obstrutiva crônica em quarto e o Diabetes em quinto lugar em 2015.

A obesidade favorece o aumento das morbimortalidades, tendo impacto importante na qualidade de vida, principalmente na população adulta, causando danos também no desenvolvimento socioeconômico do país, tendo como consequência os custos relacionados ao tratamento das complicações, hospitalizações e afastamentos do trabalho (Cembranel, et. al., 2017).

As principais comorbidades associadas à obesidade são: hipertensão arterial, doenças cardiovasculares, dislipidemia, diabetes tipo 2, síndrome de hipoventilação, apnéia do sono, osteoartrite, infertilidade, além de fatores ambientais (Mazur \& Navarro, 2015).

A modificação de alguns fatores de risco que são as dietas inadequadas, do tabagismo, pressão arterial sistólica elevada, IMC elevado, da desnutrição, atividade física insuficiente, juntamente com a implementação de programas que ampliam a assistência à saúde aos adultos, que definem metas de controle as DCNT, com ações de promoção, prevenção e assistência à saúde em seus três níveis de atenção (primário, secundário e terciário), favorecem para diminuição da obesidade, fator importante para o declínio das mortes causadas por DCNT (Souza, et. al., 2018).

Diante disso, é de extrema importância à tabulação do registro no SISVAN pelo profissional de saúde, fazendo-se necessário a avaliação nutricional periódica da população, uma vez que a partir dessas informações é possível identificar desvios nutricionais, que são fatores de risco para o desenvolvimento de DCNT, e a partir disso montar estratégias, como programas de promoção e prevenção da saúde com início na Atenção Primária, onde o enfermeiro é considerado peça importante, pois é de sua atribuição o diagnóstico situacional, com intervenção e avaliação do plano de cuidado do indivíduo e coletividade, proporcionando um aumento da expectativa de vida da população.

A falta dessas informações ou até as subnotificações expõe um cenário fictício, não mostrando a realidade, podendo trazer prejuízos irreparáveis à saúde da população a médio e longo prazo. A falta de acompanhamento periódico pelos gestores da saúde também traz consigo um declínio, pois rompe a cadeia de informação e direcionamento de recursos que é de extrema 
importância para a qualidade na prestação do serviço e atendimento direcionado para as necessidades de cada região, visto que o Brasil tem uma vasta população e muitas desigualdades sociais.

\section{Considerações Finais}

Visto que houve muitos avanços do sistema público no decorrer do tempo em busca da melhoria na qualidade da alimentação da população brasileira. Hoje encontramos novos desafios, que são o combate ao sobrepeso e a obesidade.

A Educação Permanente é uma excelente ferramenta utilizada também na Atenção Primária para a qualidade na assistência, pois é a partir dela que os profissionais de saúde adquirem o conhecimento direcionado para a sua melhor prática profissional de acordo com sua vivência cotidiana facilitando o processo de trabalho e qualificando suas orientações no âmbito individual ou coletivo.

A partir disso, espera-se que a tabulação, o acompanhamento e a intervenção nutricional dos adultos atendidos na Atenção Primária à Saúde seja contínuo para que se tenha o aumento da expectativa de vida, resultando em um envelhecimento saudável da população brasileira. Vale salientar também a importância de publicações referente a temática, para que possa assim aprimorar o embasamento científico e estratégias de intervenção não só para a comunidade científica mas bem como a população.

\section{Referências}

Abreu, E. T. F. et al., (2018). Monitoramento do estado nutricional de adultos atendidos na Atenção Básica de Saúde em Salvador- BA. Revista Saúde em Foco.

Alves, K. P. S. \& Jaime, P.C., (2014). A Política Nacional de Alimentação e Nutrição e seu diálogo com a Política Nacional de Segurança Alimentar e Nutricional. Ciência \& Saúde Coletiva, 19.

Azakura, L. et al., (2015). Fatores relacionados aos padrões alimentares de indivíduos com síndrome metabólica que frequentam ambulatório de nutrição em São Paulo. Nutrire.

Brasil. Ministério da Saúde. Secretaria de Atenção à Saúde. Departamento de Atenção Básica. (2008). Política nacional de alimentação e nutrição / Ministério da Saúde, Secretaria de Atenção à Saúde, Departamento de Atenção Básica. (2a ed.), Ministério da Saúde.

Brasil. Ministério da Saúde. Conselho Nacional de Saúde. (2012). Resolução $\mathrm{n}^{\circ} 466$, de 12 de dezembro de 2012. <https://bvsms.saude.gov.br/bvs/saudelegis/cns/2013/res0466_12_12_2012.html >.

Brasil. Ministério da Saúde. Secretaria de Atenção à Saúde. Departamento de Atenção Básica. (2013). Política Nacional de Alimentação e Nutrição. Ministério da Saúde, Secretaria de Atenção à Saúde. Departamento de Atenção Básica. Básica. Ministério da Saúde.

Brasil. Ministério da saúde. Sistema de Vigilância Alimentar e Nutricional - SISVAN. Relatórios de Acesso Público. $<$ http://sisaps.saude.gov.br/sisvan/relatoriopublico/index>.

Brasil. Portaria no $\mathrm{n}^{\circ}$ 2.488, de 21 de outubro. (2011). Aprova a Política Nacional de Atenção Básica, estabelecendo a revisão de diretrizes e normas para a organização da Atenção Básica, para a Estratégia Saúde da Família (ESF) e o Programa de Agentes Comunitários de Saúde (PACS). Diário Oficial da União 2011; 24 Out.

Brasil. Ministério da saúde. Secretaria de atenção à saúde. Departamento de atenção Básica. (2014). Guia alimentar para a população brasileira / ministério da saúde, secretaria de atenção à saúde, departamento de atenção Básica. ministério da saúde.

Brasil. Ministério da Saúde. Secretaria de Atenção à Saúde. Departamento de Atenção Básica. (2015). Marco de referência da vigilância alimentar e nutricional na atenção básica / Ministério da Saúde, Secretaria de Atenção à Saúde, Departamento de Atenção Básica. - Brasília: Ministério da Saúde.

Brasil. (2017) Plano Nacional de Segurança Alimentar e Nutricional - PLANSAN 2016-2019. MDSA, CAISAN.

Cabral, M. J., et. al., (2013). Perfil socioeconômico, nutricional e de ingestão alimentar de beneficiários do Programa Bolsa Família. Estudos Avançados.

Cavalcante, J. B., et. al., (2017). Ingestão de energia e nutrientes segundo consumo de alimentos fora do lar na Região Nordeste: uma análise do Inquérito Nacional de Alimentação 2008-2009. Rev. Bras. Epidemiologia.

Cembranel, F., et. al., (2017). Relação entre consumo alimentar de vitaminas e minerais, índice de massa corporal e circunferência da cintura: estado de base populacional com adultos no Sul do Brasil. Cad. Saúde Pública.

Development Initiatives. (2018). Global Nutrition Report: Shining a light to spur action on nutrition. Development Initiatives. 
Dutta, M., et. al., (2019). The double burden of malnutrition among adults in India: evidence from the National Family Health Survey-4 (2015-16). Epidemiol Health.

Global Burden of Disease. (2019). Health effects of dietary risks in 195 countries, 1990-2017: a systematic analysis for the Global Burden of Disease Study 2017. Lancet. 393, 1958-72.

Jaime, P. C., et al., (2018). Um olhar sobre a agenda de alimentação e nutrição nos trinta anos do Sistema Único de Saúde. Ciência \& Saúde Coletiva.

Lourenço, A. E. P., et. al., (2019). Influência da ambiência escolar no estado nutricional de pré-escolares de Macaé, Rio de Janeiro, Brasil. Ciência \& Saúde Coletiva.

Martins, J. J. (2015). Como escrever trabalhos de conclusão de curso: instruções para planejar e montar, desenvolver, concluir, redigir e a apresentar trabalhos monográficos e artigos. 9 ed. - Petrópolis, RJ: Vozes.

Mattos. A. C. E.; Rocha. L. S. \& Rodrigues. L. (2018). Diálogo sobre alimentação e nutrição na saúde mental: Ações promotoras de saúde por meio de oficinas de horticultura. RASBRAN - Revista da Associação Brasileira de Nutrição. (2), 17-24.

Mazur, C. E. \& Navarro, F. (2015). Insegurança alimentar e obesidade em adultos: Qual a relação? Saúde. 41(2).

MDS. (2018). Princípios e Práticas para Educação Alimentar e Nutricional. Ministério do Desenvolvimento Social- MDS Secretaria Nacional de Segurança Alimentar e Nutricional - SESAN.

Nascimento, F. A.; Silva, S. A. \& Jaime, P. C. (2017). Cobertura da avaliação do estado nutricional no Sistema de Vigilância Alimentar e Nutricional brasileiro: 2008 a 2013. Cadernos de Saúde Pública.

Pesquisa nacional de saúde 2019: Atenção primária à saúde e informações antropométricas: Brasil / IBGE, Coordenação de Trabalho e Rendimento. IBGE, (2020). 66p

Pesquisa de Orçamentos Familiares 2017-2018: avaliação nutricional da disponibilidade domiciliar de alimentos no Brasil / IBGE. (2020). Coordenação de Trabalhos e Rendimentos. IBGE.

Ricardi, L.M. \& Sousa, M. F. (2015). Educação permanente em alimentação e nutrição na Estratégia Saúde da Família: encontros e desencontros em municípios brasileiros de grande porte. Ciência e saúde coletiva.

Oliveira, F. A. A. \& Carvalho, F. A. F. (2020). Extinção do CONSEA ou instituição do "descontrole social" na Política Nacional de Alimentação Escolar? Jornal de Políticas Educacionais. 14(15).

Silva, E. A. et al., (2016). Excesso de pesos em usuários de Unidades Básicas de Saúde. Arquivos Brasileiros de Ciências da Saúde - ABCS Health Sci.

Silva, R. V. et al., (2019). Índice de qualidade da dieta e presença de fatores de risco para DCNTs em colaboradores de uma UAN. RASBRAN - Revista da Associação Brasileira de Nutrição. 10(2), 09-17.

Silva, V. S. et al., (2015). Correspondência entre excesso de peso e indicadores socioeconômicos e demográficos na população adulta brasileira. Revista Brasileira de Epidemiologia. 2015.

Soares, J. M. D. et al., (2020). (In)Segurança alimentar, indicadores socioeconômicos e perfil antropométrico de mulheres contempladas por programas social. Revista Brasileira de Ciência e Saúde.

Sousa, F. A. C. G. (2016). Avaliação da Atenção Básica à Saúde no Município de Jacareí. R bras ci Saúde.

Sousa, M. F. M. et. al., (2018). Transição da saúde e da doença no Brasil e nas Unidades Federadas durante os 30 anos do Sistema Único de Saúde. Ciência \& Saúde Coletiva.

UNA-SUS/UFMA. (2017). Alimentação e Nutrição na Atenção Básica em Saúde /Paola Trindade Garcia; Regimarina Soares Reis (Org.). - São Luís: EDUFMA.

Vedana, E. H. B., et al., (2008). Prevalência de Obesidade e Fatores Potencialmente Causais em Adultos em Região do Sul do Brasil. Arq Bras Endocrinol Metab.

Vigitel Brasil 2019. (2020). Vigilância de fatores de risco e proteção para doenças crônicas por inquérito não telefônico: estimativas sobre frequência e distribuição sociodemográfica de fatores de risco e proteção para doenças crônicas nas capitais dos 26 estados brasileiros e no Distrito Federal em 2019 [recurso eletrônico] / Ministério da Saúde, Secretaria de Vigilância em Saúde, Departamento de Análise em Saúde e Vigilância de Doenças não Transmissíveis. - Brasília: Ministério da Saúde.

WHO. World Health Organization. (1998). Obesity: Preventing and managing the global epidemic - Report of a WHO consultation on obesity. Geneva: WHO.

WHO. (2017). The double burden of malnutrition. Policy brief. Geneva: World Health Organization.

Zhong, Y. \& Moon, H. C. (2020). What Drives Customer Satisfaction, Loyalty, and Happiness in Fast-Food Restaurants in China? Perceived Price, Service Quality, Food Quality, Physical Environment Quality, and the Moderating Role of Gender. Foods. 